y D valores similares para partículas mayores a $3,35 \mathrm{~mm}$, a diferencia de las turbas $\mathrm{B}$ y $\mathrm{C}$ que presentaron un bajo porcentaje. El análisis de partículas mas finas (menos de $1 \mathrm{~mm}$ ) mostró que la turba $\mathrm{B}$ es la que mayor porcentaje tiene $(70,3 \%)$. Del análisis de humedad y materia seca, se destacó la turba B con un bajo valor de humedad. La densidad aparente de la turba D fue la mayor con $0,32 \mathrm{gcc}^{-1}$. El porcentaje de aire fue similar para todas las turbas, no así el porcentaje de agua, donde la turba $\mathrm{D}$ con $54,8 \%$ mostró el menor valor y la turba $\mathrm{C}$ el mayor valor con $72,9 \%$. La porosidad mayor correspondió a la turba $\mathrm{C}$ y $\mathrm{A}$. Como resultado de los análisis químico, el pH fué de 3,74 para la turba $\mathrm{D}$ y 4,34 para la turba $C$, como valores extremos. Los valores de CE fueron muy variables entre 70 microS $\mathrm{cm}^{-1}$ para la turba $\mathrm{C}$ y 730 microS$\mathrm{cm}^{-1}$ para la turba D. El análisis de los cationes mostró para la turba B los mayores valores relativos de calcio $(7,22 \mathrm{ppm})$ y magnesio $(3,34$ $\mathrm{ppm}$ ), para la turba $\mathrm{C}$ el mayor valor de potasio $(5,51 \mathrm{ppm})$ y valores similares en el nivel de sodio para todas las turbas, entre 8 y $9 \mathrm{ppm}$.

\section{CONCLUSIONES}

En Argentina se comercializan turbas con características físicas y químicas buenas, aceptables para ser usadas como sustratos de plantas ornamentales. De las turbas evaluadas, 3 (A, B y C) presentan valores de densidad aparente similares a los promedios de las turbas del Hemisferio Norte $\left(0,06-0,10 \mathrm{gcc}^{-1}\right)$, (Fonteno, 1996); sin embargo, el porcentaje de aire fue mayor al promedio $(12-20 \%)$ y el porcentaje de agua menor $(75-80 \%)$. La porosidad ha mostrado valores similares al promedio (89-94\%). Los parámetros químicos han mostrado valores similares al promedio, salvo la CE de la turba D que merece un análisis posterior.

\section{BIBLIOGRAFÍA}

Fonteno, W. C. 1996. Growing media: types and physical/chemical properties. In: Reed, E. (ed.). Water, Media and Nutrition for Greenshouse Crops. Ball Publishing pp. 93-122.

* Trabajo financiado por el Proyecto FIA-PI-C-2002-1-A-070 y el Centro Milenio de Estudios Avanzados en Ecología y Biodiversidad (CMEB, proyecto $\left.\mathrm{N}^{\circ} \mathrm{P} 02-051-\mathrm{F} \mathrm{ICM}\right)$.

Agro Sur $34(1-2): 52-532006$

\title{
DESCRIPCIÓN Y CICLO DE CRECIMIENTO DE RAICES PROTEOIDES
}

\section{DESCRIPTION AND GROWING CYCLE OF CLUSTER ROOTS}

\author{
Verdugo, G. y Zapata, K. \\ Universidad Católica de Valparaíso, Facultad de Agronomía. Casilla 4 D. Quillota. \\ E-mail: gverdugo@ucv.cl
}

\section{INTRODUCCIÓN}

Las raíces proteoides o proteiformes, son conglomerados de raicillas, que nacen muy próximas entre sí y en hileras longitudinales en torno a un eje. Deben su nombre a que son abundantemente producidas por las plantas de la familia Proteaceae. Corresponden a un sistema radicular muy particular, en Chile sólo existen en algunas especies nativas, como el avellano chileno (Gevuina avellana Mol.). Permiten a la planta crecer en suelos muy pobres, con bajas concentraciones de minerales, especialmente fósforo y otros compuestos nutritivos. Los objetivos de este trabajo fueron:

1- Describir el crecimiento de este tipo de raíces durante un período de observación de 6 meses, determinar su distribución en el perfil del suelo.

2- Apoyar la descripción de crecimiento con 
una descripción anatómica e histológica.

\section{MATERIAL Y MÉTODO}

El crecimiento radical fue medido en tres ejemplares adultos de Leucadendron "safari sunsent" (Proteaceae), establecidas al aire libre. Se establecieron tres rizotrones de $60 \mathrm{~cm}$ de profundidad por $60 \mathrm{~cm}$ de ancho y $80 \mathrm{~cm}$ de largo, a $20 \mathrm{~cm}$ del tallo. Los rizotrones fueron construidos con una pared de vidrio reforzado con marcos de madera. Después de varias semanas de instalado el rizotron se comenzó las evaluaciones mediante el marcado "in situ", que consiste en enumerar mediante trozos de mica cada una las raíces presentes, de modo que pueda ser identificado cada cambio, para ello se tomó una muestra de 20 renuevos, que medían entre $0.6 \mathrm{y}$ $0.7 \mathrm{~cm}$ de longitud. Las mediciones se realizaron cada 15 días, desde el 21 de febrero hasta el 7 de junio, fecha en que se pudo observar la última aparición de raíces.

Para una estimación de la distribución radicular, la pared de observación se dividió en 9 cuadrantes de $400 \mathrm{~cm}^{2}(20 \mathrm{~cm}$ de profundidad $\mathrm{x}$ $20 \mathrm{~cm}$ de largo). El conteo se llevó a cabo en las mismas fechas utilizando mica transparente de dimensiones equivalentes a un cuadrante, subdividida en cuadriculas de $1 \mathrm{~cm}^{2}$. El trozo de material cuadriculado fue puesto sobre cada uno de los cuadrantes ubicados en la pared, eligiendo al azar 40 cuadriculas $(10 \%$ del área de cada cuadrante) en la que se contó la cantidad de raíces presentes. Evaluaciones: Crecimiento quincenal. Densidad de raíces por $\mathrm{cm}^{2}$ promedio del número de raíces encontrado en las 40 cuadrículas de un cuadrante, como cada cuadrícula tiene un área de $1 \mathrm{~cm}^{2}$, este parámetro queda expresado en raíces $\mathrm{cm}^{2}$. Frecuencia de raíces, indica presencia o ausencia de raíces, se obtiene al dividir la cantidad de cuadrículas en la que existan una ó más raíces por el total de cuadrículas (40).

\section{RESULTADOS Y DISCUSIÓN}

Los datos revelan un máximo de actividad hacia el mes de marzo, alcanzando una tasa de crecimiento de $3.8 \mathrm{~mm}$ día-_ Se puede observar además que los valores van decreciendo paulatinamente, hasta llegar casi a cero en los primeros días de junio.

En general, las raíces siguen un patrón de crecimiento manteniéndose activa (raíz con punta blanca), hasta alcanzar aproximadamente los 35 $\mathrm{mm}$, luego suberizan tornándose de color café, deteniéndose temporalmente el proceso, para luego retomarlo alrededor de 15 días después, incrementando su longitud en los llamados "'flash" de crecimiento, claramente visibles.

Las raíces que se situaban a mayor profundidad lograron mayores longitudes y perduraron por mucho más tiempo, además su morfología era la de una raíz proteoide.

En cuanto al patrón de distribución, los primeros renuevos aparecieron a una distancia de 12 $\mathrm{cm}$ desde la superficie y en una densidad muy baja, concentrándose principalmente en los cuadrantes inferiores del perfil de observación, esto es, cuadrante 7,8 y 9 , donde fue posible encontrar en cada fecha de medición los valores más altos de las variables estudiadas.

Los más altos índices de densidad expresados en raíces $\mathrm{cm}^{-1}$, se dieron en los meses de marzo y abril, siendo de 0.51 y 0.48 respectivamente. Así mismo ocurre con la frecuencia de raíces por cuadrícula, con un valor promedio de 0.39 para el mes de marzo y 0.36 en abril.

Al integrar los datos de crecimiento radicular con los de distribución, se deduce que los períodos de elevado crecimiento coinciden con una alta emergencia de renuevos.

\section{CONCLUSIONES}

Los resultados, indican que el desarrollo de este tipo de raíces ocurre en periodos de altas temperaturas y de baja humedad, y que efectivamente presentan un mayor número de modificaciones a nivel de la epidermis, los llamados "pelos absorbentes", que cumplen el rol de absorción de agua, por tanto existiría una relación directa entre su formación y los bajos contenidos de agua. 